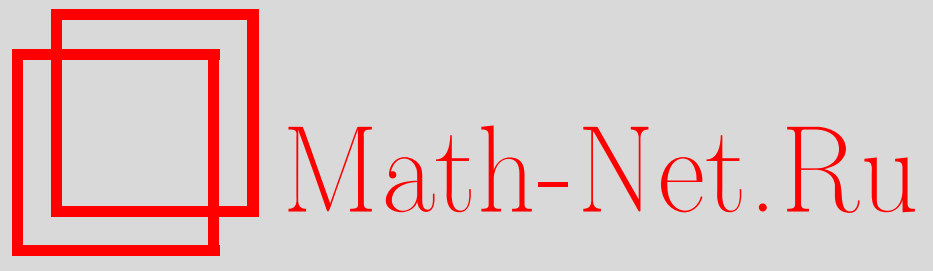

В. П. Маслов, Интегральные уравнения и фазовые переходы в вероятностных играх. Аналогия со статистической физикой, Теория вероятн. и ее примен., 2003, том 48, выпуск 2, 403-411

DOI: https://doi.org/10.4213/tvp294

Использование Общероссийского математического портала MathNet.Ru подразумевает, что вы прочитали и согласны с пользовательским соглашением

http://www . mathnet.ru/rus/agreement

Параметры загрузки:

IP : 35.173 .219 .149

26 апреля 2023 г., 13:50:28

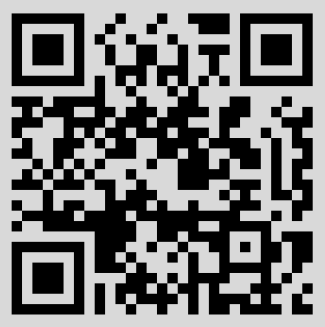


(c) 2003 г.

МАСЛОВ В. П.*

\section{ИНТЕГРАЛЬНЫЕ УРАВНЕНИЯ И ФАЗОВЫЕ ПЕРЕХОДЫ В ВЕРОЯТНОСТНЫХ ИГРАХ. АНАЛОГИЯ СО СТАТИСТИЧЕСКОЙ ФИЗИКОЙ}

Известно, что максимизация информации Кульбака-Лейблера приводит к общим преобразованиям Эшера. Статистикам Бозе-Эйнштейна и Ферми-Дирака в вероятностном пространстве $(\Omega, \mathscr{F}, P)$ отвечает другая информация, а именно

$$
S_{B}=\int \ln \left(1+\frac{d P}{d Q}\right) d Q+\int \ln \left(1+\frac{d Q}{d P}\right) d P
$$

- для бозе-статистики и

$$
S_{F}=\int \ln \left(\frac{d P}{d Q}-1\right) d Q-\int \ln \left(1-\frac{d Q}{d P}\right) d P, \quad \frac{d P}{d Q}>1,
$$

- для ферми-статистики. Она приводит к соответствующим этим статистикам преобразованиям мер. При наличии матрицы выплат эти преобразования изменяются согласно приведенным в статье интегральным уравнениям. Приводятся примеры финансовых игр, отвечающих бозе- и ферми-статистикам.

Ключевые слова и фразы: бозе-статистика, ферми-статистика, матрица выплат, преобразование Эшера, энтропия, фазовые переходы, интегральные уравнения, информация Кульбака-Лейблера, термодинамика, статистическая физика, диадические игры.

1. Преобразование вероятностных мер. В предыдущей статье автора [8] рассматривалась задача на экстремум математического ожидания при заданной энтропии. Задача решалась методом неопределенных множителей Лагранжа. В частности, было получено хорошо известное распределение Гиббса для случайной величины $X$, принимающей значения $x_{1}, \ldots, x_{n}$ :

$$
\bar{p}_{\alpha}^{(\theta)}=\frac{e^{-x_{\alpha} / \theta}}{\sum_{\beta=1}^{N} e^{-x_{\beta} / \theta}}, \quad \beta=1, \ldots, n,
$$

в случае, когда вероятности, отвечающие $x_{\alpha}$, между собой равны, а $x_{\alpha}$ несоизмеримы. Здесь $\theta$ - температура, играющая роль множителя Лагранжа (см. [8]).

Предположим теперь, что заданы частотные значения вероятностей случайной величины $X$, т.е. при $M$ испытаниях $x_{\alpha}$ выпадает $g_{\alpha}$ раз. Тогда формула Гиббса приобретает вид

$$
\bar{p}_{\alpha}^{(\theta)}=\frac{g_{\alpha} e^{-x_{\alpha} / \theta}}{\sum_{\beta=1}^{N} g_{\beta} e^{-x_{\beta} / \theta}}=\frac{p_{\alpha} e^{-x_{\alpha} / \theta}}{\sum_{\beta=1}^{N} p_{\beta} e^{-x_{\beta} / \theta}},
$$

где $p_{\alpha}=g_{\alpha} / M$ - частотная вероятность. Теперь распределение Гиббса можно записать как преобразование вероятностей

$$
\bar{P}^{(\theta)}=\frac{e^{-X / \theta}}{\Phi(\theta)} P
$$

где $\Phi(\theta)$ - нормирующий множитель.

Тривиальное обобщение этого преобразования выглядит следуюшим образом.

* Московский государственный университет им. М. В. Ломоносова, физический факультет, кафедра квантовой статистики, Ленинские горы, 119992 Москва, Россия; e-mail: victor@maslov.msk.su 
Пусть $X$ - действительная случайная величина на вероятностном пространстве $(\Omega, \mathscr{F}, P)$.

Семейство вероятностных мер $P^{(\theta)}, \theta \in \mathbf{R}$, определяемых преобразованием Гиббса, будет иметь вид:

$$
\bar{P}^{(\theta)}(d \omega)=\frac{e^{-X(\omega) / \theta}}{\Phi(\theta)} P(d \omega)
$$

где $\Phi(\theta)$ - нормирующий множитель. Его существование предполагается.

Это преобразование было введено в финансовой математике в 1935 г. и называется преобразованием Эшера [9].

П р и м е р 1. Пусть $\lambda_{n}-$ собственные значения самосопряженного оператора $\widehat{H}$ с дискретным спектром в гильбертовом пространстве $\mathscr{H}$, тогда распределение Гиббса равно $\bar{p}^{(\theta)}\left(\lambda_{n}\right)=e^{-\lambda_{n} / \theta} \sigma\left(\lambda_{n}\right) / \Phi(\theta)$, где $\sigma\left(\lambda_{n}\right)$ - кратность собственного значения $\lambda_{n}$.

Таким образом мы можем сопоставить кратность собственного значения его частотной вероятности. Однако чтобы это сопоставление было точным, нужно, чтобы $\sum_{n} \sigma\left(\lambda_{n}\right)=M<\infty$, т.е. спектр был конечный. Асимптотика при $M \rightarrow \infty, n \rightarrow \infty$, $N \rightarrow \infty$ приводит к (1), а также к (4), (5), (8), (10).

П р и м е $\mathrm{p} 2$. Пусть $\omega=(p, q), x(\omega)=H(p, q)$, где $H(p, q)$ - функция Гамильтона, $H(p, q) \in C^{\infty}\left(\mathbf{R}^{2 n}\right), p=\left(p_{1}, \ldots, p_{n}\right), q=\left(q_{1}, \ldots, q_{n}\right) ; P(d \omega)=d p d q$.

Тогда

$$
\bar{P}^{\theta}(d \omega)=\frac{e^{-H(p, q) / \theta}}{\sum} d p d q
$$

где $\sum-$ нормирующий множитель (статистическая сумма). газа.

В физике ([12], см. также [10, с. 26]) известны распределения для бозе- и ферми-

В работах [11], [8] показано, что если $G_{s}$ - кратности собственных значений одночастичного оператора Гамильтона $\widehat{H}$, то

$$
\begin{aligned}
& \Gamma_{B}=\prod_{i=1}^{n} \frac{\left(G_{i}+N_{i}-1\right) !}{\left(G_{i}-1\right) ! N_{i} !} \\
& \Gamma_{F}=\prod_{i=1}^{n} \frac{G_{i} !}{N_{i} !\left(G_{i}-N_{i}\right) !}
\end{aligned}
$$

равны кратностям собственных значений $N$-частичного оператора $\sum_{i=1}^{N} \widehat{H}_{i}$ (см. [8]) на симметрическом (соответственно антисимметрическом) по перестановке частиц подпространстве векторов (функций) $\Psi$ от $N$ частиц, т.е. от $N$ дискретных переменных. В этом случае, если определить частотные вероятности $N$ частиц как $N_{i} / N$, то при $M / N \rightarrow \rho>0$, где $M=\sum G_{i}$, для $p_{i}=\lim N_{i} / N, q_{i}=\lim G_{i} / M$ мы получим следующее значение удельной энтропии для бозе-статистики:

$$
S_{B}=\frac{1}{N} \ln \Gamma_{B} \approx \sum_{i}\left[\left(p_{i}+q_{i} \rho^{-1}\right) \ln \left(p_{i}+q_{i} \rho^{-1}\right)-p_{i} \ln p_{i}-q_{i} \rho^{-1} \ln q_{i} \rho^{-1}\right] .
$$

Аналогично удельная энтропия ферми-статистики равна

$$
S_{F}=\frac{1}{N} \ln \Gamma_{F} \approx \sum_{i}\left[q_{i} \rho^{-1} \ln q_{i} \rho^{-1}-p_{i} \ln p_{i}-\left(q_{i} \rho^{-1}-p_{i}\right) \ln \left(q_{i} \rho^{-1}-p_{i}\right)\right] .
$$

Более общее определение следуюшее.

Пусть $(\Omega, \mathscr{F}, P)$ - измеримое пространство с $\sigma$-конечной мерой $P$. Тогда

$$
S_{B}=\int \ln \left(1+\frac{d P}{d Q}\right) d Q+\int \ln \left(1+\frac{d Q}{d P}\right) d P
$$

- энтропия, отвечающая бозе-статистике,

$$
S_{F}=\int \ln \left(\frac{d P}{d Q}-1\right) d Q-\int \ln \left(1-\frac{d Q}{d P}\right) d P, \quad \frac{d P}{d Q}>1
$$


- энтропия, отвечающая ферми-статистике, при условии, что интеграл от модулей подынтегральных выражений меньше бесконечности.

Отсюда аналогично [12] получаем

$$
\bar{P}_{\alpha}^{(\theta)}=\frac{1}{e^{X_{\alpha} / \theta} \Phi(\theta, \rho)-\rho} P_{\alpha}
$$

где $\Phi(\theta, \rho)$ - нормирующий множитель, для бозе-систем, и

$$
\bar{P}_{\alpha}^{(\theta)}=\frac{1}{e^{X_{\alpha} / \theta} \Phi(\theta, \rho)+\rho} P_{\alpha}
$$

для ферми-систем, где множитель $\Phi(\theta, \rho)$ также находится из условия нормировки. Его существование также предполагается.

Приведем по аналогии с формулой (1) преобразование вероятностных мер.

Пусть снова $X$ - действительная случайная величина на вероятностном пространстве $(\Omega, \mathscr{F}, P)$.

Семейство вероятностных мер $P^{(\theta)}, \theta \in \mathbf{R}$, определяемых преобразованием для бозе-статистик, будет иметь вид:

$$
\bar{P}^{(\theta)}(d \omega)=\frac{1}{e^{X(\omega) / \theta} \Phi(\theta, \rho)-\rho} P(d \omega) .
$$

П р и м е р 3. Пусть $\lambda_{n}$ - собственные значения самосопряженного оператора $\widehat{H}$ с дискретным спектром в гильбертовом пространстве $\mathscr{H}$, тогда распределение Бозе может быть записано следующим образом:

$$
\bar{P}^{(\theta)}\left(\lambda_{n}\right)=\frac{\sigma\left(\lambda_{n}\right)}{e^{\lambda_{n} / \theta} \Phi(\theta, \rho)-\rho}
$$

где $\sigma\left(\lambda_{n}\right)$ - кратность собственного значения $\lambda_{n}$.

П р и м р р 4. Пусть $\omega=(p, q), x=H(p, q), P(d \omega)=d p d q, \theta=k T$, где $H(p, q)$ некоторая функция Гамильтона, $p \in \mathbf{R}^{n}, q \in \mathbf{R}^{n}$, тогда преобразование (8) приобретет следующий вид:

$$
\bar{P}^{(\theta)}(d \omega)=\frac{d p d q}{\exp \{H(p, q) /(k T)\} \Phi(k T, \rho)-\rho}
$$

Аналогично определяется семейство вероятностных мер $\bar{P}^{(\theta)}, \theta \in \mathbf{R}$, соответствующее преобразованию (7) для ферми-статистики:

$$
\bar{P}^{(\theta)}(d \omega)=\frac{1}{e^{X(\omega) / \theta} \Phi(\theta, \rho)+\rho} P(d \omega) .
$$

П р и м е $\mathrm{p}$. Пусть снова $\lambda_{n}-$ собственные значения самосопряженного оператора $\widehat{H}$, а $\sigma\left(\lambda_{n}\right)$ - их кратности, тогда распределение Ферми (7) приобретает вид

$$
\bar{P}^{(\theta)}\left(\lambda_{n}\right)=\frac{\sigma\left(\lambda_{n}\right)}{e^{\lambda_{n} / \theta} \Phi(\theta, \rho)+\rho} .
$$

П р и м е р 6. Пусть $\omega=(p, q), P(d \omega)=d p d q, \theta=k T, x=H(p, q)$, где $H(p, q)$ некоторая функция Гамильтона, $p \in \mathbf{R}^{n}, q \in \mathbf{R}^{n}$, тогда преобразование (10) приобретет следующий вид:

$$
\bar{P}^{(\theta)}(d \omega)=\frac{d p d q}{\exp \{H(p, q) /(k T)\} \Phi(k T, \rho)+\rho} .
$$

Отметим, что последние распределения не рассматриваются в классической статистической физике, но они имеют место при переходе из квантовой статистики при $h \rightarrow 0$. Этот факт впервые был замечен автором (см. [13]). Тем не менее физики фактически применяли его для ферми-систем в температурном уравнении Томаса-Ферми [14]. Оно будет приведено ниже. 
2. Матрица выплат и интегральные уравнения. Пусть $p_{1}, \ldots, p_{n}-$ вероятности того, что случайная величина $X$ принимает значения $x_{1}, \ldots, x_{n}$ соответственно, и пусть $\bar{p}_{1}, \ldots, \bar{p}_{n}$ - преобразование этих вероятностей, зависящее от $x_{1}, \ldots, x_{n}$ :

$$
\bar{p}_{1}=f_{1}\left(x_{1}, \ldots, x_{n}, \theta\right) p_{1}, \ldots, \bar{p}_{n}=f_{n}\left(x_{1}, \ldots, x_{n}, \theta\right) p_{n} .
$$

Например, преобразование (12) является преобразованием Гиббса-Эшера, БозеЭйнштейна-Эшера или Ферми-Дирака-Эшера.

Введем матрицу выплат $\left\{a_{i k}\right\}_{i=1, \ldots, n, k=1, \ldots, n}$, зависящую от пары компонент вектора $\left(x_{1}, \ldots, x_{n}\right)$ : каждой паре $x_{i}, x_{k}$ мы ставим в соответствие «выплату» $a_{i k}$ (термин заимствован из теории игр).

Например, в игре в орлянку есть только два значения случайной величины и если дважды выпал орел, то платится налог на выигрыш в размере $a_{11}$, если дважды выпала решка, то платится дотация $a_{22}$, если первый раз выпал орел, а второй решка или наоборот, то платится налог держателю игорного дома - $a_{12}$.

Если играют $N$ игроков, то все $a_{i j}$ делятся на $N$, т.е. каждая пара, в которой один получил $x_{i}$, а другой $-x_{j}$, независимо от того, в каком месте пространства они находятся, выплачивает дополнительный налог $a_{i j} / N$.

При этом каждый игрок звонит каждому другому, они сравнивают случайные «выигрыши» (у одного, например, $x_{i}$, у другого $-x_{j}$ ) и получают компенсацию или выплачивают налог $a_{i j}$.

Описано, таким образом, парное взаимодействие. В отличие от модели Изинга, где «опрашиваются» только соседи, в этой игре соседей нет. Аналогом является газ частиц, каждая из которых взаимодействует с каждой, 一 парное взаимодействие. Оно в нашем случае «глобальное» (но малое: $\sim 1 / N$ ) в отличие от модели Изинга, где взаимодействие локально. В физике в этом случае говорят о дальнодействии. Однако в модели газа, состоящего из твердых шариков, взаимодействие короткодействующее, хотя «соседом» может оказаться любая частица, в отличие от «кристаллических», пронумерованных элементов в модели Изинга. Этот термин связан с пространственной переменной, которая в нашей «игровой» модели отсутствует.

Мы определили преобразования Гиббса-Эшера, Бозе-Эйнштейна-Эшера и Ферми-Дирака-Эшера для невзаимодействующих игроков. Теперь определим, как будет меняться распределение, если учитывать малое, $\sim 1 / N$, парное взаимодействие или уплату по матрице выплат.

Приведем уравнение, которому будет удовлетворять это преобразование при $N \rightarrow \infty$. Рассмотрим уравнение для $\bar{x}_{i}: \bar{x}_{i}=x_{i}+\sum_{j=1}^{n} a_{i j} f_{j}\left(\bar{x}_{1}, \ldots, \bar{x}_{n}, \theta\right) p_{j}$, из которого находятся так называемые «одетые» $\bar{x}_{i}$, и искомое преобразование имеет вид: $\bar{p}_{j}=f_{j}\left(\bar{x}_{1}, \ldots, \bar{x}_{n}, \theta\right) p_{j}$. Это преобразование (распределение) может иметь особенности типа фазовых переходов, подобные тем, которые возникают в модели Изинга.

Приведем обобщение этого уравнения на уже рассмотренный случай $(1),(6),(9)$. В этом случае вместо матрицы выплат мы рассмотрим оператор выплат $A(\omega, \bar{\omega})$, где $A(\omega, \bar{\omega})$ - функция, измеримая по паре $\omega, \bar{\omega}$.

Для классической статистики получаем уравнение

$$
\bar{X}(\omega)=X(\omega)+\int_{\Omega} A(\omega, \bar{\omega}) \frac{e^{-\bar{X}(\bar{\omega}) / \theta}}{\Phi(\theta)} P(d \bar{\omega}) .
$$

Для объектов, не меняющихся при перестановке (бозе-случай), -

$$
\bar{X}(\omega)=X(\omega)+\int_{\Omega} A(\omega, \bar{\omega}) \frac{P(d \bar{\omega})}{e^{\bar{X}(\bar{\omega}) / \theta} \Phi(\theta, \rho)-\rho},
$$

и для объектов, отвечающих статистике Ферми, -

$$
\bar{X}(\omega)=X(\omega)+\int_{\Omega} A(\omega, \bar{\omega}) \frac{P(d \bar{\omega})}{e^{\bar{X}(\bar{\omega}) / \theta} \Phi(\theta, \rho)+\rho} .
$$

Полученные «одетые» решения $X(\omega)$ подставляются в формулы $(1),(6),(9)$, что дает соответственно распределение для трех статистик. 
П р и м е р 7. Уравнение Больцмана-Пуассона-Власова для «одетого» потенциала будет иметь вид (при $\bar{p}=p)$ :

$$
\frac{\overline{p^{2}}}{2 m}+\bar{u}(q)=\frac{p^{2}}{2 m}+u(q)+\int \frac{V\left(q-q^{\prime}\right) e^{-\left(p^{\prime 2} /(2 m)+u\left(q^{\prime}\right)\right) / \theta} d p^{\prime} d q^{\prime}}{\int e^{-\left(p^{\prime 2} /(2 m)+u\left(q^{\prime}\right)\right) / \theta} d p^{\prime} d q^{\prime}} .
$$

Здесь гамильтониан $H(p, q)=p^{2} /(2 m)+u(q), m$ - масса, а $V\left(q-q^{\prime}\right)$ - потенциал взаимодействия между частицами.

Уравнения для «одетых» потенциалов в случае ферми- и бозе-распределений соответственно имеют вид:

$$
\bar{u}(q)=u(q)+\int \frac{V\left(q-q^{\prime}\right) d p^{\prime} d q^{\prime}}{\exp \left\{\theta^{-1}\left(\left|p^{\prime}\right|^{2} /(2 m)+\bar{u}\left(q^{\prime}\right)\right)\right\} \Phi(\theta, \rho)+\rho}
$$

(температурное уравнение Томаса-Ферми, $[14$, с. 339]) и

$$
\bar{u}(q)=u(q)+\int \frac{V\left(q-q^{\prime}\right) d p^{\prime} d q^{\prime}}{\exp \left\{\theta^{-1}\left(\left|p^{\prime}\right|^{2} /(2 m)+\bar{u}\left(q^{\prime}\right)\right)\right\} \Phi(\theta, \rho)-\rho} .
$$

Последнее уравнение не было известно в физике. Его впервые получил автор в [15] - для случая $\theta=0$ и в [7] (формула (6)) - для общего случая. Бифуркация решений приведенных выше интегральных уравнений приводит к фазовым переходам [16]-[18].

3. Точно решаемые примеры вероятностных игр. Сравнение решения уравнения «одетого» гамильтониана с точным решением. Есть различие в определении энтропии в физике, с одной стороны, и в теории информации и финансовой математике - с другой. Они отличаются знаком.

П р и м е р 8. Пусть $N$ игроков играют в орла и решку с банком. Если у них есть возможность перевернуть монету в выгодное для них положение, то они придут к выигрышу, равному $N \alpha$, где $\alpha$ - плата за выпадение одного орла.

В теории вероятностей и моделях рынка [19] есть целый ряд марковских процессов - так называемое глауберово движение, hot-bath - «горячая ванна», Mегаполис и др., когда постепенно мы приходим к распределению Гиббса. В работе [2] (гл. $2, \S 2.9)$ показано, что конечный результат будет один и тот же; значит, огрубляя процесс, зависяший от времени, ограничиваясь простым переворачиванием монеты, мы приходим к тому же результату. Таким образом, данную задачу можно рассматривать как задачу на выигрыш, но с дополнительным налогом, который равен температуре (или волатильности), умноженной на энтропию Шеннона. Это можно интерпретировать как налог на «неточность информации».

Теперь предположим, что каждый играющий связывается по телефону с каждым другим и если у обоих выпала решка, то они получают одинаковую дотацию из банка, равную $2 V / N$. Отсюда следует, что всего банк выплачивает

$$
\frac{2 N_{1}\left(N_{1}-1\right)}{N} \sim 2 n^{2} N
$$

где $N \gg 1, n=N_{1} / N$, а $N_{1}$ - число выпадений решки.

Поэтому общий выигрыш, деленный на $\alpha N$, будет равен

$$
f(n, \theta)=1-n+2 V n^{2}-\theta n \ln n-\theta(1-n) \ln (1-n) .
$$

Предположим, что $V=1$ и результат исходного броска всеми играющими таков, что $n$ меньше того значения $n_{0}(\theta)$, которое дает минимум для выигрыша (13). Если температура $\theta$ меньше некоторой $\theta_{c}$, а каждый переворот монеты приводит к большему выигрышу, то в результате выигрыш будет равен меньшему из максимальных значений $f(n, \theta)$.

Это значит, что при малом и медленном изменении температуры игроки переворачивают мало монет, а при $\theta>\theta_{c}$ для того, чтобы выиграть, они должны переворачивать сразу большое количество монет.

Если речь идет не об игре в орла-решку, а о покупке-продаже акций, то такой скачок означает «пробой» по терминологии рынка. 
П р и м е р 9. Бозе-статистика. Многогранные кости. Мы приведем здесь пример точно решаемой вероятностной игры, связанной с энтропией бозеэйнштейновского типа, и покажем на этом примере, что она может решаться с помощью уравнения (11).

Мы рассмотрим следующую игру. Одновременно кидают $N$ одинаковых костей, каждая кость имеет $M$ граней, грани с номером $i$ соответствует число $\lambda_{i}, \lambda_{i}<\lambda_{i+1}$, $\lambda_{i}<0$ при $i \leqslant G_{1}, \lambda_{i}>0$ при $i>G_{1}$. Выигрыш (доход) вычисляется следующим образом. Обозначим через $n_{i}$ число костей, у которых выпала грань с номером $i$. Очевидно, что $\sum_{i=1}^{M} n_{i}=N$. Пусть $G_{1}, G_{2}>0$ - целые числа такие, что $G_{1}+G_{2}=M$. Введем для броска одной кости осредненный проигрыш

$$
\varepsilon_{1}=\frac{1}{G_{1}} \sum_{i=1}^{G_{1}} \lambda_{i}
$$

и выигрыш

$$
\varepsilon_{2}=\frac{1}{G_{2}} \sum_{i=1}^{G_{2}} \lambda_{G_{1}+i},
$$

тогда выигрыш для броска $N$ костей задается формулой

$$
\mathscr{E}=\varepsilon_{1} N_{1}+\varepsilon_{2} N_{2},
$$

где $N_{1}, N_{2}$ выражаются следуюшим образом: $N_{1}=\sum_{i=1}^{G_{1}} n_{i}, N_{2}=\sum_{i=1}^{G_{2}} n_{i+G_{1}}$. Очевидно, что $N_{1}+N_{2}=N$. Будем считать, что кости являются неразличимыми. В таком случае число возможных вариантов бросания $N$ костей с выигрышем $\mathscr{E}=\varepsilon_{1} N_{1}+\varepsilon_{2} N_{2}$ равно

$$
\Gamma\left(N_{1}, N_{2}\right)=\sum_{K_{1}=0}^{N} \cdots \sum_{K_{M}=0}^{N} \delta_{L_{1} N_{1}} \delta_{L_{2} N_{2}},
$$

где $\delta_{L N}$ - символ Кронекера, а $L_{1}, L_{2}$ зависят от $K_{1}, \ldots, K_{M}$ следующим образом: $L_{1}=\sum_{i=1}^{G_{1}} K_{i}, L_{2}=\sum_{i=1}^{G_{2}} K_{i+G_{1}}$. Легко убедиться, что (14) может быть записано в виде:

$$
\Gamma\left(N_{1}, N_{2}\right)=\frac{\left(N_{1}+G_{1}-1\right) !}{N_{1} !\left(G_{1}-1\right) !} \cdot \frac{\left(N_{2}+G_{2}-1\right) !}{N_{2} !\left(G_{2}-1\right) !} .
$$

Введем энтропию, отвечающую выигрышу $\mathscr{E}=\varepsilon_{1} N_{1}+\varepsilon_{2} N_{2}$, следуюшим образом:

$$
S\left(N_{1}, N_{2}\right)=\ln \left(\Gamma\left(N_{1}, N_{2}\right)\right) .
$$

Кроме того, будем считать, что в игре взимается налог на выигрыш. А именно, у каждой пары выигравших костей - таких костей, у которых выпала грань с номером $i>G_{1}$, - отнимается величина $V / N, \varepsilon_{2}-\varepsilon_{1}<V<2\left(\varepsilon_{2}-\varepsilon_{1}\right)$. Кроме того, компенсируемый налог равен $\theta S\left(N_{1}, N_{2}\right)$, где $\theta$ - константа. Поскольку таких пар всего $N_{2}\left(N_{2}-1\right) / 2$ штук, то окончательный выигрыш (доход) в игре составит

$$
\mathscr{E}\left(N_{1}, N_{2}\right)=\varepsilon_{1} N_{1}+\varepsilon_{2} N_{2}-\frac{V N_{2}\left(N_{2}-1\right)}{2 N}-\theta S\left(N_{1}, N_{2}\right) .
$$

В отличие от обычных игр на выигрыш, мы рассмотрим игру в поддавки, чтобы оставалась прямая аналогия с физикой. Каждый играющий старается проиграть как можно больше, скажем, если сумма выигрыша вычитается из жалования, которое он получает.

Каждому играющему предоставляется право, увидев ответ, присоединиться к $\varepsilon_{1}$ или $\varepsilon_{2}$ по его желанию, т.е. поменять $N_{1}$ на $N \pm 1$ и $N_{2}$ на $N_{2} \pm 1$, выбирая знак + или -, так, чтобы выигрыш уменьшился. Нетрудно видеть, что если $N_{2}^{0}$, при котором достигается $\max \mathscr{E}\left(N-N_{2}, N_{2}\right)$, не лежит на конце интервала $0 \leqslant N_{2} / N \leqslant 1$, то когда в игре выпало $N_{2}<N_{2}^{0}$, игроки будут вычитать из $N_{2}$ единицу, а когда $N_{2}>N_{2}^{0}-$ прибавлять. В результате можно прийти к одному из минимумов: глобальному или локальному. Если увеличивать параметр $\theta$, то при некотором $\theta_{0}$ максимум и локальный минимум сольются и при $\theta>\theta_{0}$ будет лишь один глобальный минимум. Если 
игроки находились на локальном минимуме, то, значит, часть из них должна будет быстро присоединяться к другой группе, чтобы попасть на глобальный минимум.

В нашем случае $h(x)=\lambda(x)+\sum_{x^{\prime}} V\left(x, x^{\prime}\right)$, где $\lambda(x)=\varepsilon_{1}$ при $1 / M \leqslant x \leqslant G_{1} / M$ и $\lambda(x)=\varepsilon_{2}$ при $G_{1} \leqslant x \leqslant 1, V\left(x, x^{\prime}\right)=-V$ при $\left(G_{1}+1\right) / M \leqslant x \leqslant 1$ и $\left(G_{1}+1\right) / M \leqslant x^{\prime} \leqslant 1$ и $V\left(x, x^{\prime}\right)=0$ вне этих интервалов.

Уравнение для «одетого» гамильтониана $\widetilde{h}(x)$ в данном случае имеет вид:

$$
\widetilde{h}(x)=\lambda(x)+\frac{1}{N} \sum_{x^{\prime}=1}^{M} V\left(x, x^{\prime}\right) f\left(\widetilde{h}\left(x^{\prime}\right)\right),
$$

где функция $f(h), h \in \mathbf{R}$, имеет вид $f(h)=(\exp \{(h-\mu) / \theta\}-1)^{-1}$. Здесь $\theta-$ множитель, а $\mu$ - нормирующий множитель, который находится из условия $N=$ $\sum_{x=1}^{M} f(\widetilde{h}(x))$. Отсюда следует, что решение уравнения (18) имеет вид $\widetilde{h}(x)=h_{1}$ при $1 \leqslant x \leqslant G_{1} / M, \widetilde{h}(x)=h_{2}$ при $\left(G_{1}+1\right) / M \leqslant x \leqslant M$, где $h_{1}=\varepsilon_{1}$, а $h_{2}$ определяется из уравнения

$$
h_{2}=\varepsilon_{2}-\frac{g_{2} V}{\rho} f\left(h_{2}\right), \quad g_{i}=\frac{G_{i}}{N},
$$

здесь $G_{2}=M-G_{1}$. Уравнение (18) для множителя $\mu$ примет вид $\rho=g_{1} f\left(h_{1}\right)+g_{2} f\left(h_{2}\right)$. Мы получим систему уравнений

$$
\begin{gathered}
\rho=\frac{g_{1}}{\exp \left\{\left(\varepsilon_{1}-\mu\right) / \theta\right\}-1}+\frac{g_{2}}{\exp \left\{\left(h_{2}-\mu\right) / \theta\right\}-1}, \\
h_{2}=\varepsilon_{2}-\frac{g_{2} V}{\rho} \frac{1}{\exp \left\{\left(h_{2}-\mu\right) / \theta\right\}-1} .
\end{gathered}
$$

Величины $\mu$ и $h_{2}$ определяются из уравнений (19), (20). Кроме того, они удовлетворяют условиям $\varepsilon_{1}-\mu>0, h_{2}-\mu>0$, а $\varepsilon_{1}, \varepsilon_{2}, V, \theta$ удовлетворяют условиям

$$
\varepsilon_{2}-\varepsilon_{1}>0, \quad \varepsilon_{2}-\varepsilon_{1}-V<0, \quad \theta>0 .
$$

Выразим множитель $\mu$ из уравнения (20): $\mu=h_{2}-\theta \ln \left(1+g_{2} V / \rho\left(\varepsilon_{2}-h_{2}\right)\right)$. Bведем $n_{1}, n_{2}$ следуюшим образом:

$$
n_{1}=\frac{1}{\exp \left\{\left(\varepsilon_{1}-\mu\right) / \theta\right\}-1}, \quad n_{2}=\frac{1}{\exp \left\{\left(h_{2}-\mu\right) / \theta\right\}-1} .
$$

Из (21) следует, что $n_{1}>0, n_{2}>0$.

Уравнения (19), (20) соответственно примут вид:

$$
n_{1}=\frac{\rho-g_{2} n_{2}}{g_{1}}, \quad h_{2}=\varepsilon_{2}-\frac{g_{2} V}{\rho} n_{2} .
$$

Кроме того,

$$
\mu=\varepsilon_{1}+\theta \ln \left(\frac{n_{1}}{1+n_{1}}\right), \quad \mu=h_{2}+\theta \ln \left(\frac{n_{2}}{1+n_{2}}\right) .
$$

Приравнивая правые части уравнений (23) и подставляя выражения (22), получим следуюшее уравнение для $n=q_{2} n_{2} / \rho, \varepsilon=\varepsilon_{2}-\varepsilon_{1}$ :

$$
\varepsilon-V n+\theta \ln \left(\frac{n}{g_{2}+n} \cdot \frac{g_{1}+1-n}{1-n}\right)=0 .
$$

Если в (17) перейти к пределу при $N \rightarrow \infty, N / M \rightarrow \rho$ с помощью формулы Стирлинга, то, полагая $N_{1} / N \rightarrow n_{1}, N_{2} / N \rightarrow n_{2}$ и приравнивая производную по $n_{2}\left(n_{1}=1-n_{2}\right)$ к нулю, получим второе уравнение (22).

П р и м е р 10. Ферми-статистика. Размещение по очередям. Пример взят из «Двенадцати стульев». Альхен посылал опекаемых им старушек в очереди за сахаром, который выдавали по $\frac{1}{2}$ килограмма на человека, чтобы потом продать сахар по спекулятивной цене. Пусть имеются две очереди: в одной продукт дает после продажи «навар» $\varepsilon_{1}$, а в другой $-\varepsilon_{2}$, причем $\varepsilon_{2}>\varepsilon_{1}$, но чтобы попасть во вторую 
очередь, взимается взятка с каждой пары старушек, равная $V / N$, где $N$ - число старушек. Пусть одна очередь длины $G_{1}$, а вторая длины $G_{2}, G_{1}+G_{2}=M>N$. Так как старушки становятся обязательно на разные места очереди, то число возможных размешений равно $\Gamma_{F}$ при $n=2$ (формула (3)) и мы приходим аналогично предыдушему примеру к формуле (15), где $S=\ln \Gamma_{F}$. Мы получим в результате то же уравнение $(16)$, где $f(h)=(\exp \{h / \theta\} \Phi(\theta, \rho)+\rho)^{-1}$.

Предположим, что $N$ старушек наугад разошлись по двум магазинам, а далее стали свободно переходить из одного в другой с целью уменьшения при каждом переходе дохода Альхена, которого не любили. Мы попадаем в ту же ситуацию, что и в предыдушем примере. Если $M / N$ достаточно велико, то при некотором $\theta_{0}$ локальный минимум и глобальный максимум сольются и при $\theta>\theta_{0}$ старушки должны будут дружно ринуться в другую очередь.

Во всех трех примерах этого пункта скачок не является аналогом фазового перехода первого рода (и тем более второго, третьего и т.д. рода). Здесь «скачет» свободная энергия. Этот переход аналогичен переходу термодинамики движущейся сверхтекучей жидкости (терминология автора, см. [6], [20], [21]) в обычную термодинамику. И в этом смысле скачок аналогичен эффекту фонтанирования, полученному экспериментально в 1938 г. Д. Алленом и Х. Джонсом [22], [23, гл. Х].

Автор выражает глубокую благодарность А. С. Черному за ценные замечания.

\section{СПИСОК ЛИТЕРАТУРЫ}

1. Bucklew J. Large Deviation Technique in Decision, Simulation, and Estimation. New York: Wiley, 1990, $270 \mathrm{p}$.

2. Cover T. M., Thomas J. A. Elements of Information Theory. New York: Wiley, 1991, $542 \mathrm{p}$.

3. Csiszar I. I-divergence geometry of probability distributions and minimization problems. - Ann. Probab., 1975, v. 3, № 1, p. 146-158.

4. Föllmer H., Schied A. Stochastic Finance. An Introduction in Discrete Time. Berlin: de Gruyter, 2002, 422 p.

5. Frittelli $M$. The minimal entropy martingale measure and the valuation problem in incomplete markets. - Math. Finance, 2000, v. 10, № 1, p. 39-52.

6. Маслов В. П. Квантование термодинамики и ультравторичное квантование. М.: Институт компьютерных исследований, 2001, $384 \mathrm{c.}$

7. Maslov V.P. Quasi-particles associated with Lagrangian manifolds corresponding to classical self-consistent fields. I-XII.-Russian J. Math. Phys., 1994, v. 2, p. 527-534; 1995 , v. 3, p. 123-132, 271-276, 401-406, 529-534; 1996, v. 4, p. 117-122, 265-270, $539-546 ; 1997 / 98$, v. 5 , p. 123-130, 273-278, 405-412, 529-534.

8. Маслов В.П. Методы квантовой статистики с точки зрения теории вероятностей. - Теория вероятн. и ее примен., 2002, т. 47, в. 4, с. 686-709.

9. Ширяев $A . H$. Основы стохастической финансовой математики. Т. 2: Теория. М.: ФАЗИС, $1998,544 \mathrm{c}$.

10. Ширяев А.Н. Вероятность. М.: Наука, 1989,640 с.

11. Маслов В.П. Эконофизика и квантовая статистика. - Матем. заметки, 2002, т. 72 , № 6, c. 883-891.

12. Ландау Л. Д., Лившич Е. М. Статистическая физика. М.: Наука, 1964.

13. Maslov V.P. On a new class of integral equations with jumping nonlinearity. - Math. Res., 1994 , v. 82 , p. $217-225$.

14. Ашкрофт Н., Мермин $Н$. Физика твердого тела. Т. 1. М.: Мир, 1979.

15. Маслов $B$. П. Теорема о классическом пределе $\hbar \rightarrow 0$ для $N$ взаимодействуюших фермионов. - Матем. заметки, 1998, т. 63, № 1, с. 145-146.

16. Маслов В.П. О фазовом переходе для классических фермионов. - Матем. заметки, 1998 , т. 63 , № 4, с. 635-637.

17. Маслов В. П. О фазовом переходе для классических бозонов, фермионов и обычных классических частиц. - Матем. заметки, 1998, т. 63, № 5, с. 792-795. 
18. Маслов В. П. О фазовом переходе для классических фермионов. - Матем. заметки, 1998, т. 64, № 3, с. 470-473.

19. Blume L. E. The statistical mechanics of strategic interaction. - Games and Economic Behavior, 1993, v. 5, № 3, p. 387-424.

20. Маслов В.П. Двухуровневая модель слабо неидеального бозе-газа. Фазовый переход в метастабильном (сверхтекучем) состоянии. - Докл. РАН, 2003, т. 389, № 4, c. 1-2.

21. Маслов В.П. Модель слабо неидеального бозе-газа. Фазовый переход в сверхтекучем состоянии и эффект фонтанирования. - Вестник МГУ, сер. физ., астрон., 2003, № 1, с. 3-11.

22. Кеезом В. Гелий. М., 1949.

23. Физика низких температур. М.: ИЛ, 1959.

Поступила в редакцию 17.III. 2003

(c) 2003 г

МАТИЯСЕВИЧ Ю. В.*

\section{ОДИН ВЕРОЯТНОСТНЫЙ ЭКВИВАЛЕНТ ГИПОТЕЗЫ ЧЕТЫРЕХ КРАСОК}

Для каждого плоского тривалентного графа естественным образом строится вероятностное пространство и определяются два случайных события; гипотеза четырех красок оказывается эквивалентной (положительной) корреляции этих событий.

Ключевые слова и фразы: гипотеза четырех красок.

В 2002 году знаменитой гипотезе четырех красок исполнилось 150 лет. Согласно исследователям истории математики (см., например, [8], [18]), единоличное авторство этой гипотезы принадлежит Френсису Гатри (Francis Guthrie). В 1852 году он раскрашивал карту Англии и обнаружил, что достаточно использовать всего четыре краски для того, чтобы раскрасить всю карту так, чтобы любые два соседних английских графства были окрашены в разные цвета. Френсис предположил, что четырех красок хватило бы и для любой мыслимой карты (разумеется, раскрашиваемые области должны быть связными). Не будучи в состоянии доказать это, Френсис обратился за помощью к своему брату - студенту-математику Фредерику (Frederick Guthrie). Tот в свою очередь адресовал этот вопрос своему профессору де Моргану (A. de Morgan), который стал задавать этот вопрос другим.

Первое «доказательство» гипотезы Гатри было опубликовано А.Б. Кемпе [15] четверть века спустя; еще через десятилетие в нем была найдена ошибка.

Кемпе стал первым, но не последним автором ошибочного доказательства гипотезы Гатри. Ситуация изменилась в 1976 году, когда К. Аппель и В. Хакен [2] объявили о доказательстве гипотезы о четырех красках, полученном с помощью интенсивного счета на компьютерах. Объем потребовавшихся вычислений был столь велик, что найденное доказательство [3], [4] не могло быть проверено человеком.

Сейчас, четверть века спустя, положение дел существенно не изменилось. К. Аппель и В. Хакен опубликовали новое, несколько более простое доказательство [5]. Н. Робертсон, Д. Сандерс, П. Сеймур и Р. Томас, придя к выводу, что они не могут проверить в этом доказательстве даже ту часть, которая была сделана без компьютеров, написали новые программы и получили дальнейшие упрощения. Тем не менее

* С.-Петербургское отделение Математического института им. В. А. Стеклова PAH, Фонтанка, 27, 191011 С.-Петербург, Россия; URL: http://logic.pdmi.ras.ru/ ${ }^{\sim}$ yumat 